\title{
Dyons near the transition temperature in $S U(3)$ lattice gluodynamics
}

\author{
V. G. Bornyakov \\ Institute for High Energy Physics NRC "Kurchatov Institute", 142281 Protvino, Russia, \\ Institute of Theoretical and Experimental Physics, 117259 Moscow, Russia \\ School of Biomedicine, Far East Federal University, 690950 Vladivostok, Russia \\ E.-M. Ilgenfritz \\ Joint Institute for Nuclear Research, BLTP, 141980 Dubna, Russia \\ B. V. Martemyanov \\ Institute of Theoretical and Experimental Physics, 117259 Moscow, Russia \\ National Research Nuclear University MEPhI, 115409, Moscow, Russia \\ Moscow Institute of Physics and Technology, 141700, Dolgoprudny, Moscow Region, Russia
}

(Dated: August 9, 2021)

\begin{abstract}
We study the topological structure of $S U(3)$ lattice gluodynamics by cluster analysis. This methodological study is meant as preparation for full QCD. The topological charge density is becoming visible in the process of overimproved gradient flow, which is monitored by means of the the Inverse Participation Ratio (IPR). The flow is stopped at the moment when calorons dissociate into dyons due to the overimproved character of the underlying action. This gives the possibility to simultaneously detect all three dyonic constituents of KvBLL calorons in the gluonic field. The behaviour of the average Polyakov loop under (overimproved) gradient flow could be also (as its value) a diagnostics for the actual phase the configuration is belonging to. Timelike Abelian monopole currents and specific patterns of the local Polyakov loop are correlated with the topological clusters.

The spectrum of reconstructed cluster charges $Q_{c l}$ corresponds to the phases. It is scattered around $Q_{c l} \approx \pm 1 / 3$ in the confined phase, whereas it is $Q_{c l} \approx \pm 0.5 \div 0.7$ for heavy dyons and $\left|Q_{c l}\right|<0.3$ for light dyons in the deconfined phase. We estimate the density of heavy and light dyons at three values of temperature. We find that heavy dyons are increasingly suppressed with increasing temperature.
\end{abstract}

The paper is dedicated to the memory of Michael Müller-Preussker who was a member of our research group for more than twenty years.

PACS numbers: 11.15.Ha, 12.38.Gc, 12.38.Aw

Keywords: Lattice gauge theory, caloron, dyon

\section{INTRODUCTION}

There are topological objects in the vacuum of Yang Mills theory and QCD (at finite temperature) known as constituent "dyons" of Kraan-van Baal-Lee$\mathrm{Lu}$ (KvBLL) calorons [1]. These objects have been studied in $S U(2)$ and $S U(3)$ lattice gluodynamics and then in lattice QCD both in the confined and deconfined phases [4] 8 . At the beginning one or the other method of smoothing of the lattice gauge field to get rid of the ultraviolet fluctuations was used in these studies. The fermionic topological charge density constructed from low lying modes of the overlap Dirac operator (for two or three types of temporal boundary conditions imposed on the fermion field) was more recently used for this goal. Methods connected to the spectrum of the overlap Dirac operator are rather computer time consuming. For this reason we recollect here, first for the example of pure gluodynamics, how much information in this respect can be obtained by means of purely gluonic observables: the gluonic topological charge density, the Polyakov loop (global and local) and Abelian monopoles (obtained in the Maximal Abelian Gauge 9]). Many of these tools have been proposed and used by us occasionally in the past.

The main problem with the gluonic topological charge density is that it is too noisy when measured on the vacuum lattice gluonic fields (in Monte Carlo or Hybrid Monte Carlo ensembles) such that a topological structure is not discernible. The objects we are looking for (calorons, dissociated or not dissociated into dyons) appear only in the process of cooling or its continuous analog - the gradient flow [10 12, usually realized with respect to the Wilson action (therefore Wilson flow). The gradient flow with respect to so-called over-improved action [13 has an additional built-in feature of forced dissociation of calorons into dyons [14. This is an advantage if we want to analyze the gauge field configurations down to the caloronconstituents - the dyons. During this process, the dissociation of calorons can be controlled by monitoring the inverse participation ratio (IPR) of the modulus $|q(x)|$ of the topological charge density. This IPR varies between the extreme values: IPR $=1$ (for a homogeneously delocalized density) and IPR $=V_{4}$ (for a pointlike localized density). $V_{4}$ is the four dimensional volume. The IPR is defined (analogously to the IPR 
of the scalar density $|\psi(x)|^{2}$ of fermionic modes) as follows:

$$
\mathrm{IPR}=V_{4} \frac{\sum_{x}|q(x)|^{2}}{\left(\sum_{x}|q(x)|\right)^{2}}
$$

The IPR increases when ultraviolet fluctuations of the gluonic field are removed. It also increases when already existing topological structures are removed by annihilation e.g. calorons and anticalorons as well as their dyon and antidyon constituents. At the same time the dissociation of calorons into dyons leads to a decrease of the IPR. So, we are in the position to stop the process of over-improved gradient flow (monitoring the IPR) when calorons are maximally delocalized (dissociated to dyons). We found that, as a rule, the IPR of a given configuration first grows to a maximum. Then it decreases before it grows again.

We expect that maximal delocalization (dissociation) of the calorons should happen (approximately) at the first minimum after the first maximum of the IPR in the over-improved gradient flow history. In practice we used a fixed flow time for all flow histories (at the same temperature) when they typically go through the above mentioned minimum.

Let us note that the dyons, or quark instantons, are playing the decisive role in a recent model of the QCD vacuum proposed by Shuryak and collaborators [15 19]. This model serves to expain the intermediate temperature dependence of the topological suceptibility before the onset of the Dilute Gas Approximation (DIGA). The lattice computation of the density of dyons is important for a cross-check of this model.

\section{THERMAL ENSEMBLES}

For this methodical study of dyon detection in $S U(3)$ gauge theory we employ quenched ensembles which are generated with the standard Wilson action $S_{W}$ for the lattice coupling $\beta=6 / g_{0}^{2}$.

To fix the corresponding lattice spacing $a$ as a function of $\beta$ for this action we rely on the Necco-Sommer parametrization [20].

The test ensembles (to describe three temperatures) were generated on the asymmetric lattices with a fourdimensional volume $V_{4}=a^{4} L_{t} \cdot L_{s}^{3}$, where $L_{t}=6$ is the number of sites in the temporal direction, and $L_{s}=24$ the size in all spatial directions. The phase transition for $L_{t}=6$ takes place at $\beta_{c}=5.894$ [21]. It corresponds to a critical temperature of pure gluodynamics $T_{c} \simeq 300 \mathrm{MeV}[22$.

Our test ensembles (100 configurations each ) for the three temperatures of interest, $T=0.79 T_{c}, T=$ $1.27 T_{c}$ and $T=1.5 T_{c}$, were generated with $\beta=5.8$, $\beta=6.0$ and $\beta=6.084$, respectively.

\section{FLOW HISTORIES}

For illustration, over-improved gradient flow histories for 12 configurations taken out of two ensembles, below and above $T_{c}\left(T=0.79 T_{c}\right.$ and $\left.T=1.27 T_{c}\right)$, are shown in Fig. 1 and Fig. 2, respectively. The figures show in parallel the localization of the topological density IPR, the total topological charge $Q$, and the action in instanton action units (from top to bottom) Additionally in Fig. 3 the flow histories of the volume-averaged Polyakov loop (PL) are presented. In all cases 600 steps (with step length $\epsilon=0.02$ ) of flow are shown.

The flow histories for the volume-averaged Polyakov loop are different in the confining and deconfining phases: while in the confining phase PL has no prefered direction of evolution (interpreted in 23] as indication of an equal number of dyons of all three types), in the deconfining phase PL goes in the direction where rare heavy dyons - left from supposedly asymmetric caloron solutions - become more and more heavy, whereas more abundant light dyons become more and more light (also this interpretation was given in [23]).

The flow histories for the topological charge show very fast stabilization towards integer topological charges ( up to $Q= \pm 8$ ) in the confining phase and towards $Q=0$ or $Q= \pm 1$ in the deconfining phase. In the confining phase the characteristic flow history for IPR is as follows: IPRs first grows, then decreases until - at several hundreds of flow steps - it goes through a local minimum, before finally it grows again. In the deconfining phase, IPRs always monotonously grows, for $Q= \pm 1$. For $Q=0$ configurations, the IPR typically experiences no sizable changes under gradient flow. In all cases, the action monotonously decreases, although it most rapidly does so at $T>T_{c}$.

Like in our previous paper [23], we will interpret also the IPR flow histories in terms of a dyonic picture. The growth of IPR in the confined phase can be understood as result of two processes: the removal of perturbative fluctuations and of the decreasing of the number of separate topological objects in the process of annihilation of calorons and anticalorons (as well as of their constituent dyons and antidyons). The topological charge density becomes more localized when perturbative fluctuations are hidden under the peaks of the topological charge density of topological objects. The subsequent drop of IPR we may interpret as the result of an increasing of the number of separate topological objects in the process of dissociation of calorons into constituent dyons. Thus, the first minimum (after passing the first maximum) in the history curve of IPR can be considered as a point of maximal dissociation of calorons into dyons in the course of over-improved gradient flow. We believe, it is a good choice to stop the flow process here for the observation of these dyons. As it can be seen from Fig. 1 for IPR, 

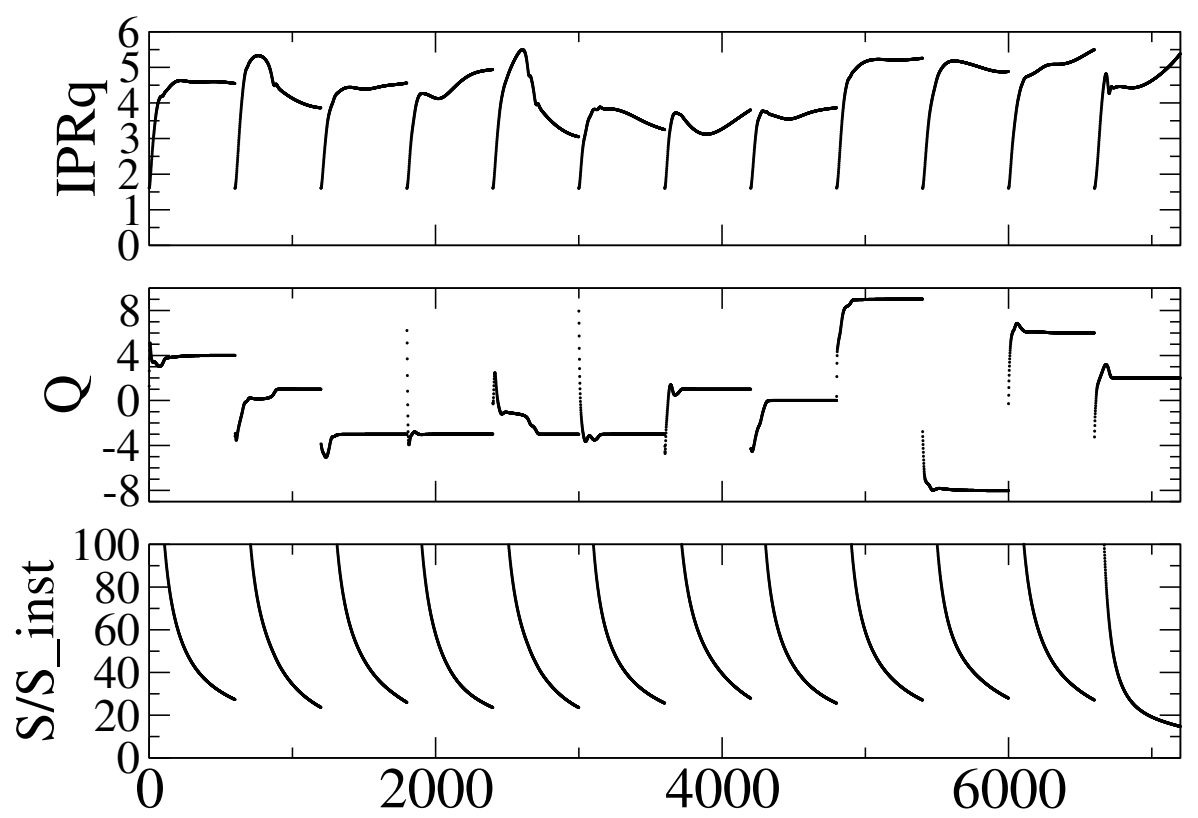

FIG. 1: Over-improved flow histories for 12 configurations below $T_{c}\left(T=0.79 T_{c}\right)$ shown for IPR, the topological charge $Q$ and the action in instanton units (from top to bottom). On X-axes the cumulative number of flow steps is shown (for every configuration 600 flow steps).
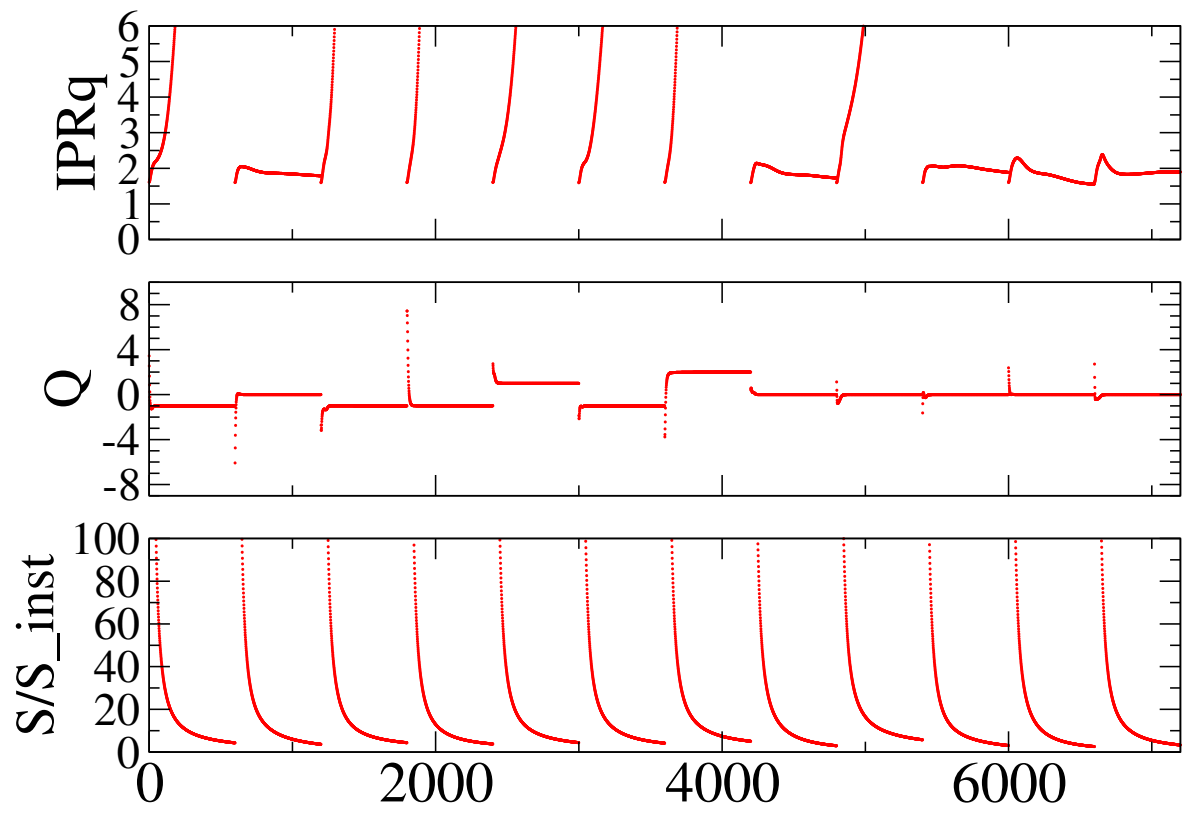

FIG. 2: Over-improved flow histories for 12 configurations above $T_{c}\left(T=1.27 T_{c}\right)$ shown for IPR, the topological charge $Q$ and the action in instanton units (from top to bottom). On X-axes the cumulative number of flow steps is shown (for every configuration 600 flow steps).

in the confined phase several hundreds of flow steps is needed to reach this point. In practice we used 600 steps to be sure that maximal dissociation has been achieved for all confining configurations.

In the deconfined phase, the topological objects expected are heavy dyons with highly localized and highvalued topological charge density on one hand and light dyons with delocalized and low-valued topological charge density on the other. The calorons are expected to be already dissociated into these asymmetric kinds of dyons. For $Q=0$ configurations in the deconfined phase we have no heavy dyons, and the IPR will experience no sizable changes during the flow process. For $Q= \pm 1$, the final IPR is defined 
a)

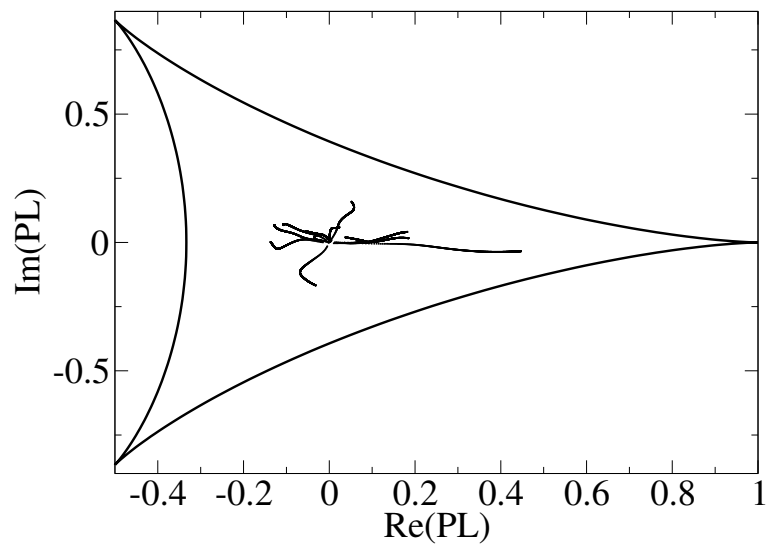

b)

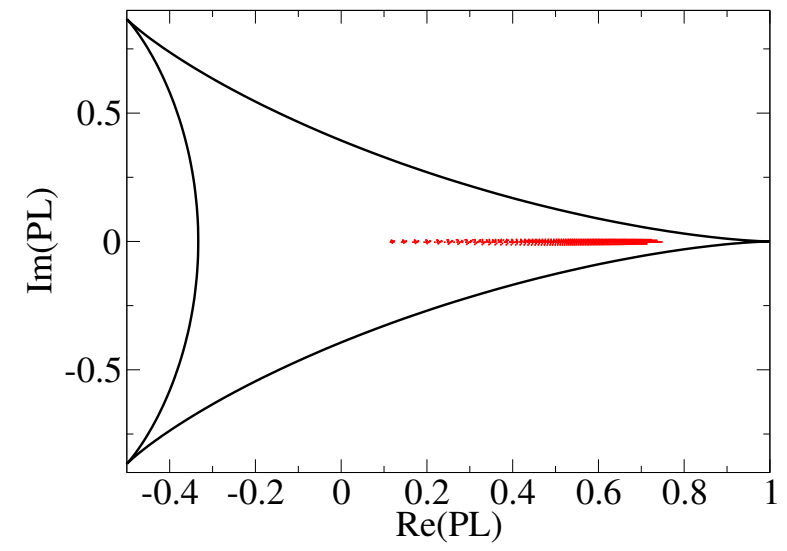

FIG. 3: Over-improved flow histories of the volume-averaged Polyakov loop for 12 configurations below/above $T_{c}$ : (a) at $T=0.79 T_{c}$ and (b) at $T=1.27 T_{c}$, respectively.

(after removal of perturbative fluctuations) by a single heavy dyon with highly localized charge density and its absolute topological charge being close to one.

Since we have no stopping criterium for the deconfined phase that would be motivated by the IPR history (opposite to the confined phase) we finish the flow process after approximately 100 steps of gradient flow when the action of a configurations is comparable with that after 600 steps of flow in the confined phase.

\section{CORRELATION BETWEEN ABELIAN MONOPOLES AND POLYAKOV LOOPS IN AFTER-FLOW GLUON FIELDS}

Relatively isolated dyons in KvBLL caloron solutions are monopoles, and the local holonomies (untraced Polyakov loops) have the specific property that two eigenvalues of the holonomy become degenerate there [24]. In the case of $S U(2)$ gauge theory this corresponds to the points where Polyakov loops take the values $\pm I$. Abelian monopoles (in the sense of Abelian projection after fixing MAG) and dyon constituents of KvBLL caloron solution (placed on the lattice) are in one-to-one correspondence (at least for isolated objects), and in the $S U(2)$ case they are correlated with the above mentioned points [25, 26].

In the $S U(3)$ case the degeneracy of Polyakov loops corresponds to the flanks of the unitarity triangle [24]. For a Polyakov loop

$$
\operatorname{diag}\left(\mathrm{e}^{2 \pi i \mu_{1}}, \mathrm{e}^{2 \pi i \mu_{2}}, \mathrm{e}^{2 \pi i \mu_{3}}\right)
$$

(with $\mu_{1} \leq \mu_{2} \leq \mu_{3} \leq \mu_{4}=1+\mu_{1}$ and $\mu_{1}+\mu_{2}+\mu_{3}=$ 0) parametrised by three numbers $m_{1}=\mu_{2}-\mu_{1}, m_{2}=$ $\mu_{3}-\mu_{2}$ and $m_{3}=\mu_{4}-\mu_{3}$ this degeneracy happens when either $m_{1}$ or $m_{2}$ or $m_{3}$ vanish.
The inter-correlation of Abelian monopoles and the monopoles defined by degenerated eigenvalues of holonomy can be detected by a correlation between loci of minimum of $m_{1}, m_{2}, m_{3}$ with Abelian monopoles localized in MAG [8]. Which one of $m_{1}, m_{2}$ or $m_{3}$ becomes very small determines the type of dyon. In Fig. 4 the distributions of $\min \left(\mathrm{m}_{1}(\mathrm{x}), \mathrm{m}_{2}(\mathrm{x}), \mathrm{m}_{3}(\mathrm{x})\right)$ taken over all lattice sites (shaded histogram) is compared with the distribution over all cubes which are duals of time-like monopole links (open red histogram). The latter are loci where thermal monopoles are located. This is shown for $T=0.79 T_{c}$ ) in the confined phase (Fig. $4 \mathrm{a}$ ) and for $T=1.27 T_{c}$ in the deconfined phase (Fig. $4 \mathrm{~b}$ ). In the case of a monopole the minimum $\min \left(\mathrm{m}_{1}(\mathrm{x}), \mathrm{m}_{2}(\mathrm{x}), \mathrm{m}_{3}(\mathrm{x})\right)$ is taken over the eight corners of the three-dimensional cube containing that monopole. It is seen that the distibution of $\min \left(\mathrm{m}_{1}(\mathrm{x}), \mathrm{m}_{2}(\mathrm{x}), \mathrm{m}_{3}(\mathrm{x})\right)$ on thermal monopoles is shifted towards zero compared with the distribution in the bulk. It is also seen that in the deconfined phase, when the Polyakov loop averaged over volume is near the corner of the unitarity triangle $((1,0)$ in our case) the distibution of $\min \left(\mathrm{m}_{1}(\mathrm{x}), \mathrm{m}_{2}(\mathrm{x}), \mathrm{m}_{3}(\mathrm{x})\right)$ on thermal monopoles which now correspond to light dyons has only minor shift in compariosn with the distribution in the bulk.

\section{CLUSTER ANALYSIS}

An access to the topological charge density at all lattice points at a selected time of the gradient flow, allows to perform a cluster analysis of the topological density, with hope to associate the emerging clusters with dyons. The method of the analysis was described many times, for example in 8. As it can 

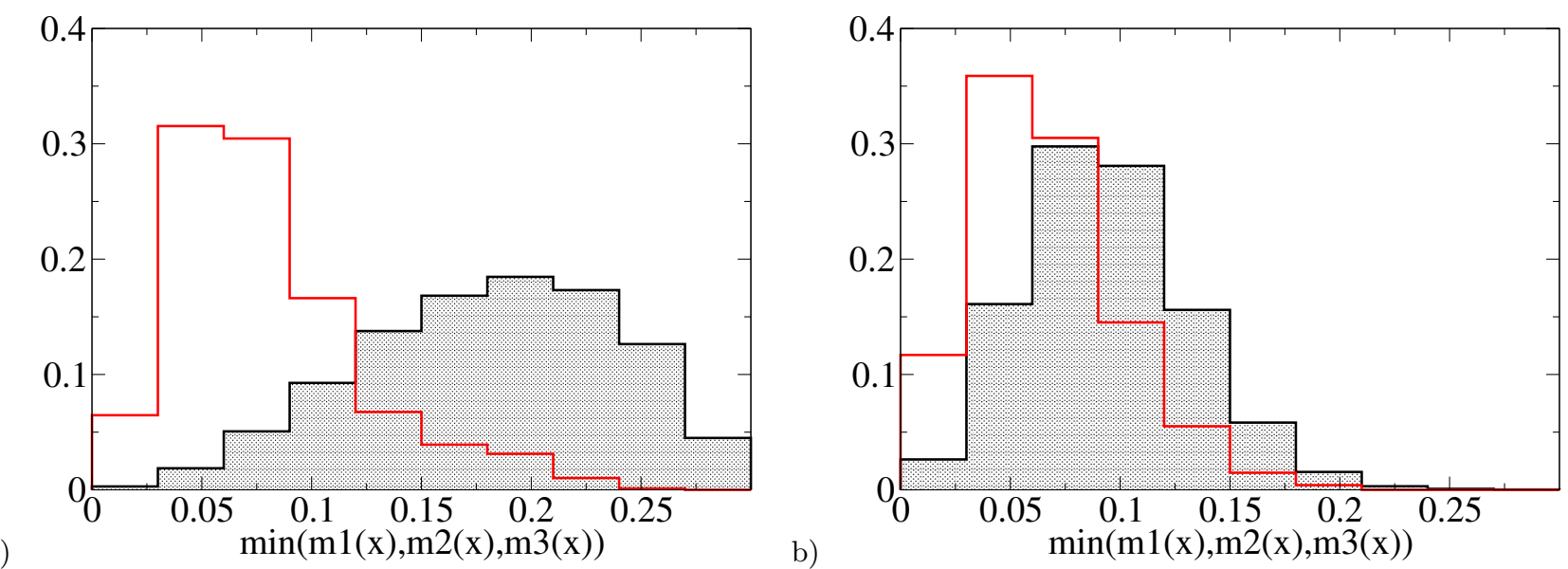

FIG. 4: The distributions with respect to the minimal distance $\min \left(\mathrm{m}_{1}(\mathrm{x}), \mathrm{m}_{2}(\mathrm{x}), \mathrm{m}_{3}(\mathrm{x})\right)$ between the local Polyakov loop value and the nearest flank of the Polyakov triangle over all lattice sites (shaded histogram) and for all cubes where thermal monopoles are located (open red histogram) are shown in (a) for $T=0.79 T_{c}$ in the confined phase and in (b) for $T=1.27 T_{c}$ in the deconfined phase.

\begin{tabular}{|l|l|l|c|c|c|c|c|c|}
\hline phase & $V_{c l}$ & $V_{\text {clmon }}$ & $N_{c l}$ & $N_{\text {clmon }}$ & $N_{\text {mon }}$ & $N_{\text {moncl }}$ & $N_{\text {loop }}$ & $N_{\text {loopcl }}$ \\
\hline$T=0.79 T_{c}<T_{c}$ & $2.6(1) \%$ & $2.4(1) \%$ & $15.4(2)$ & $10.4(2)$ & $306(6)$ & $94(3)$ & $45(1)$ & $19(1)$ \\
\hline$T=1.27 T_{c}>T_{c}$ & $2.7(3) \%$ & $1.0(1) \%$ & $29(2)$ & $3.0(2)$ & $130(3)$ & $21(2)$ & $21(1)$ & $5.2(4)$ \\
\hline$T=1.5 T_{c}>T_{c}$ & $4.7(3) \%$ & $1.2(1) \%$ & $45(2)$ & $3.0(2)$ & $106(3)$ & $19(1)$ & $18(1)$ & $5.9(4)$ \\
\hline
\end{tabular}

TABLE I: Results of the cluster analysis. All numbers indicate averages per configuration. The pure statistical errors are given in parentheses. We denote with $V_{\mathrm{cl}}$ - the volume fraction occupied by all topological clusters, $V_{\mathrm{cl}}$ mon - the volume fraction occupied by clusters containing time-like magnetic monopoles, $N_{\mathrm{cl}}$ - the number of all clusters per configuration, $N_{\mathrm{cl} \text { mon }}$ - the number of clusters containing time-like magnetic monopoles, $N_{\text {mon }}$ - the overall number of dual time-like

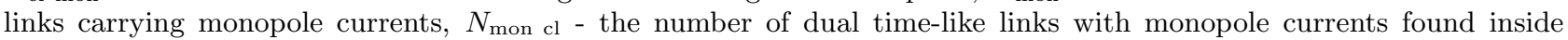
topological clusters, $N_{\text {loop }}$ - the overall number of thermal monopoles (closed in time direction magnetic current loops) , $N_{\text {loop cl }}$ - the number of these thermal monopoles piercing topological clusters.

be seen from table I, clusters occupy several percent of the lattice volume (equal in physical units to $26 \mathrm{fm}^{4}, 5 \mathrm{fm}^{4}, 2.5 \mathrm{fm}^{4}$ for ensembles at temperatures $T=0.79 T_{c}, T=1.27 T_{c}, T=1.5 T_{c}$, respectively) and are correlated to magnetic monopoles. The correlation is strongest in the confined phase.

We see that at $T=0.79 T_{c}<T_{c}$ approximately 100 (from around 300 in total) of dual time-like links carrying monopole currents are concentrated in only $2.4 \%$ of the lattice occupied by topological clusters. On the other hand the remaining approximately 200 of dual time-like links carrying monopole currents are spreaded over $97.6 \%$ of the rest of the lattice. Thus, time-like Abelian magnetic currents are about 20 times more dense inside clusters of topological charge than outside. Also, in this phase around 10 clusters containing time-like magnetic monopoles at all, are pierced totally by around $20=2 \times 10$ time-like magnetic current loops (of thermal monopoles) as it should be for a KvBLL dyon. This is because each dyon is a monopole in one Abelian field and an antimonopole in another Abelian field, for the three Abelian fields available in the case of $S U(3)$.

The (reconstructed) topological charges $\left(Q_{\mathrm{cl}}\right)$ of the clusters containing time-like magnetic monopoles are distibuted around $\pm 1 / 3$ in the confined phase (see Fig. $5 \mathrm{~b})$ ). In the deconfined phase, these clusters form two groups, one of heavy clusters with $0.5<|Q|<0.7$ on one hand and another of light clusters with $|Q|<$ 0.3 (see Fig. 6b)) on the other hand.

The "reconstructed" cluster charge is obtained by summing over the charge density according to a procedure described in [8, 25, 26], where also the systematic error is estimated. Finally, in the confined phase, the trace of the Polyakov loop measured inside the clusters in representative points (where the mini$\operatorname{mum} \min \left(\mathrm{m}_{1}(\mathrm{x}), \mathrm{m}_{2}(\mathrm{x}), \mathrm{m}_{3}(\mathrm{x})\right)$ is taken on) is indeed located closely to the flanks of the Polyakov triangle. This is an expected feature of KvBLL monopoledyons appearing in three types (of approximately 
equal charge and abundance) in the confined phase (see Fig. 5a)). The three flanks correspond to the three types of dyon observed.

\begin{tabular}{|l|l|l|c|}
\hline phase & $\rho_{3}(1)$ & $\rho_{3}(2)$ & $\rho_{3}(3)$ \\
\hline$T=0.79 T_{c}<T_{c}$ & $1.22(2)$ & $1.22(2)$ & $1.22(2)$ \\
\hline$T=1.27 T_{c}>T_{c}$ & $1.15(7)$ & $1.35(7)$ & $1.35(7)$ \\
\hline$T=1.5 T_{c}>T_{c}$ & $0.54(4)$ & $3.1(2)$ & $3.1(2)$ \\
\hline
\end{tabular}

TABLE II: $3 \mathrm{~d}$ densities $\rho_{3}(i), i=1,2,3$ for heavy, light, light dyons at all three temperatures in $1 / \mathrm{fm}^{3}$ units.

In the deconfined phase, the position of heavy and light clusters (represented by the trace of the Polyakov loop) in the Polyakov triangle is indicated by points with $\min \left(\mathrm{m}_{3}(\mathrm{x})\right)$ or $\min \left(\mathrm{m}_{1}(\mathrm{x}), \mathrm{m}_{2}(\mathrm{x})\right)$ for heavy or light clusters (see Fig. 6a)), respectively. We see that 11 out of 38 clusters found in (totally) 12 configurations are heavy so that heavy dyons are relatively suppressed with respect to the light ones. This is also an expected feature of KvBLL monopole-dyons. Finally, we can calculate $3 \mathrm{~d}$ densities for dyons at all three temperatures (for dyons as static objects $3 \mathrm{~d}$ densities are more relevant than $4 \mathrm{~d}$ densities) The suppression of heavy dyons with the increase of temperature is clearly seen (see Table II).

\section{CONCLUSIONS}

We have studied the topological structure of $S U(3)$ gluodynamics by cluster analysis of the gluonic topological density. The gluonic topological charge density was emerging in the process of gradient flow with respect to the over-improved action. Monitoring the IPR of the modulus of the topological density has allowed us to stop the gradient flow at the moment when calorons have dissociated into dyons due to overimproved character of this process. This has given us the possibility to visualize all three dyon constitutents of a KvBLL caloron formed in the gluonic field. The time-like Abelian monopoles and the specific KvBLL pattern of the local holonomy (untraced Polyakov loop) are correlated to topological clusters. The reconstructed (summed) values of topological charges for each kind of dyons are concentrated near $1 / 3$ in the confined phase. In the deconfined phase, however, the values of the cluster charges (characterizing heavy and light dyons) have been found correlated with the local holonomy. The suppression of heavy dyons with the increase of temperature is clearly seen.

\section{Acknowledgments}

V.G.B. and B.V.M. are supported by the RFBR grant 16-02-01146a. B.V.M. appreciates the support by the grants RFBR 15-02-07596a.
[1] T. C. Kraan and P. van Baal, Nucl.Phys. B533, 627 (1998), hep-th/9805168.

[2] T. C. Kraan and P. van Baal, Phys.Lett. B435, 389 (1998), hep-th/9806034.

[3] K.-M. Lee and C.-H. Lu, Phys.Rev. D58, 025011 (1998), hep-th/9802108.

[4] V. Bornyakov, E.-M. Ilgenfritz, B. Martemyanov, S. Morozov, M. Müller-Preussker, and A. Veselov, Phys.Rev. D76, 054505 (2007), 0706.4206.

[5] V. Bornyakov, E.-M. Ilgenfritz, B. Martemyanov, and M. Müller-Preussker, Phys.Rev. D79, 034506 (2009), 0809.2142 .

[6] E.-M. Ilgenfritz, B. Martemyanov, and M. MüllerPreussker, Phys.Rev. D89, 054503 (2014), 1309.7850.

[7] V. G. Bornyakov, E. M. Ilgenfritz, B. V. Martemyanov, and M. Muller-Preussker, Phys. Rev. D91, 074505 (2015), 1410.4632.

[8] V. G. Bornyakov, E. M. Ilgenfritz, B. V. Martemyanov, and M. Mller-Preussker, Phys. Rev. D93, 074508 (2016), 1512.03217.

[9] A. S. Kronfeld, G. Schierholz, and U. J. Wiese, Nucl. Phys. B293, 461 (1987).

[10] M. Luscher, Commun. Math. Phys. 293, 899 (2010), 0907.5491.

[11] M. Lüscher, JHEP 1008, 071 (2010), 1006.4518.

[12] M. Luscher and P. Weisz, JHEP 02, 051 (2011),
1101.0963.

[13] M. Garcia Perez, A. Gonzalez-Arroyo, J. R. Snippe, and P. van Baal, Nucl.Phys. B413, 535 (1994), heplat/9309009.

[14] F. Bruckmann, E.-M. Ilgenfritz, B. Martemyanov, and P. van Baal, Phys.Rev. D70, 105013 (2004), heplat/0408004.

[15] E. Shuryak, J. Phys. G39, 054001 (2012), 1112.2573.

[16] P. Faccioli and E. Shuryak, Phys. Rev. D87, 074009 (2013), 1301.2523.

[17] R. Larsen and E. Shuryak, Phys. Rev. D92, 094022 (2015), 1504.03341.

[18] R. Larsen and E. Shuryak, Nucl. Phys. A950, 110 (2016), 1408.6563.

[19] R. Larsen and E. Shuryak, Phys. Rev. D96, 034508 (2017), 1705.04707.

[20] S. Necco and R. Sommer, Phys.Lett. B523, 135 (2001), hep-ph/0109093.

[21] Y. Iwasaki, K. Kanaya, T. Yoshie, T. Hoshino, T. Shirakawa, Y. Oyanagi, S. Ichii, and T. Kawai, Phys. Rev. D46, 4657 (1992).

[22] C. Gattringer, R. Hoffmann, and S. Schaefer, Phys.Lett. B535, 358 (2002), hep-lat/0203013.

[23] V. Bornyakov, E.-M. Ilgenfritz, B. Martemyanov, V. Mitrjushkin, and M. Müller-Preussker, Phys.Rev. D87, 114508 (2013), 1304.0935. 


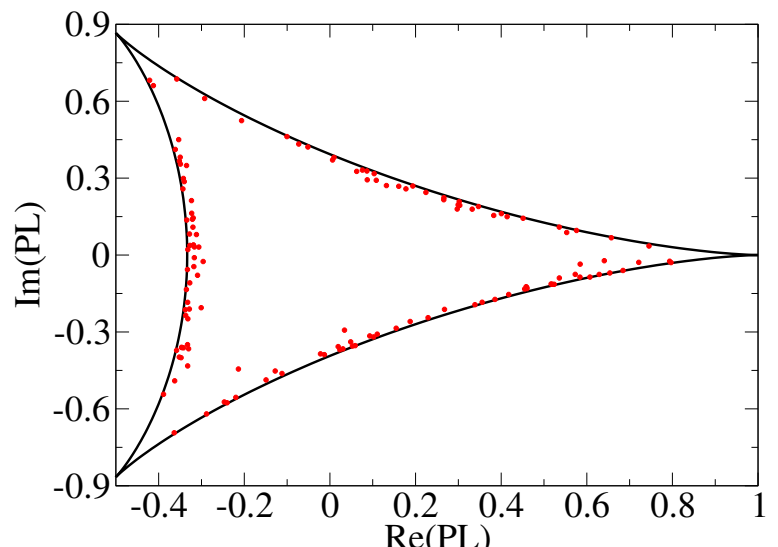

a)

b)

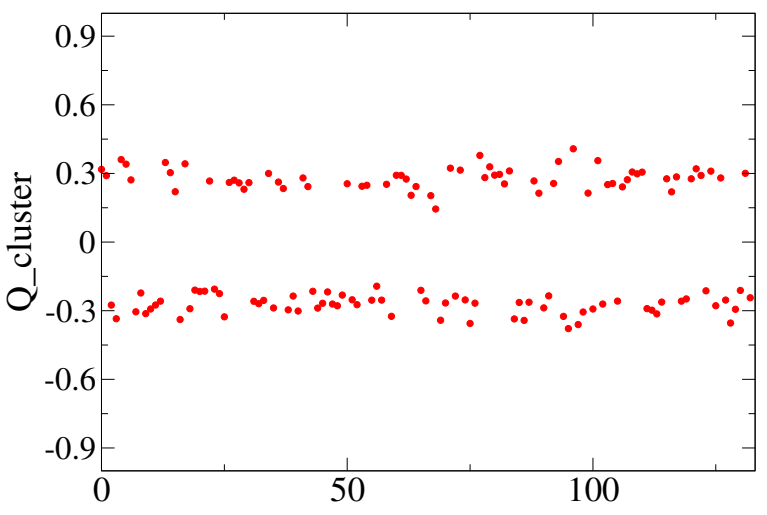

FIG. 5: a) Scatter plot of the Polyakov loop values at points with $\min \left(\mathrm{m}_{1}(\mathrm{x}), \mathrm{m}_{2}(\mathrm{x}), \mathrm{m}_{3}(\mathrm{x})\right)$ for clusters in the confined phase at $T=0.79 T_{c}$ (shown as circles, only clusters containing thermal monopoles are shown), b) integrated topological charges of these clusters shown also by circles. Abscissa here shows the number of cluster and for 12 presented configurations there are $10.4 * 12 \approx 125$ such clusters.

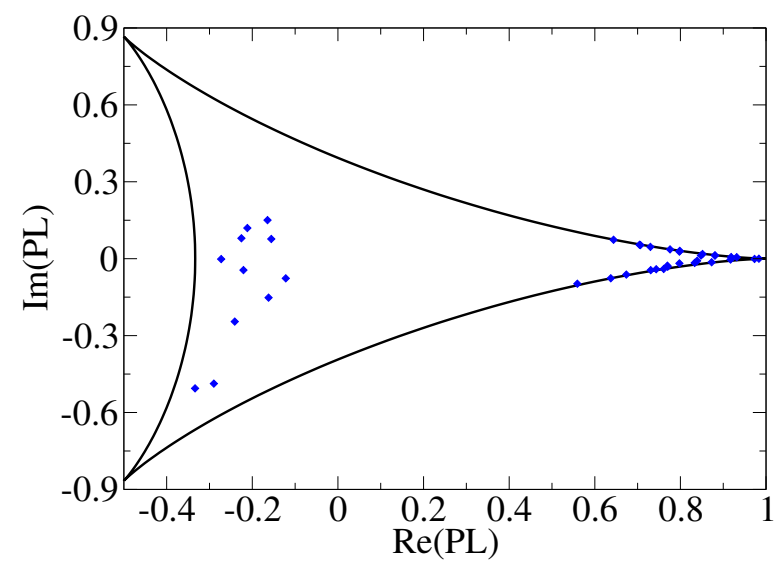

b)

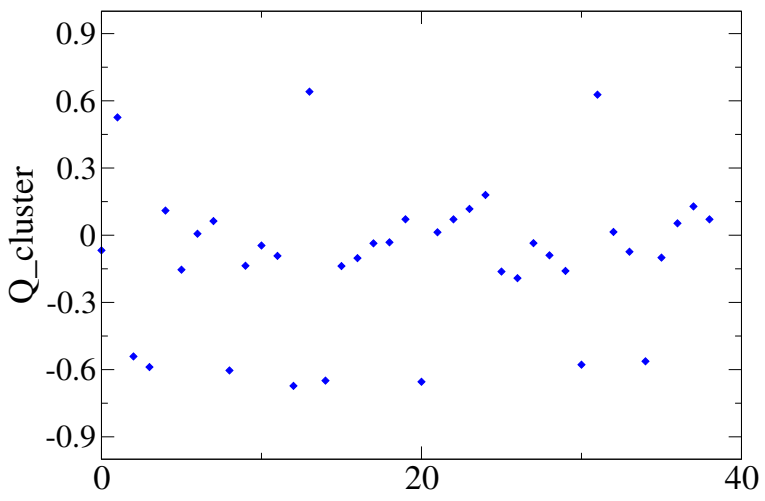

FIG. 6: a) Scatter plot of the Polyakov loop values at points with $\min \left(\mathrm{m}_{1}(\mathrm{x}), \mathrm{m}_{2}(\mathrm{x}), \mathrm{m}_{3}(\mathrm{x})\right)$ for clusters in the deconfined phase at $T=1.27 T_{c}$ (shown as diamonds, only clusters containing thermal monopoles are shown), b) integrated topological charges of these clusters shown also by diamonds. As in Fig. 5 abscissa shows the number of cluster and for 12 presented configurations there are $3.2 * 12 \approx 38$ such clusters.

[24] P. Van Baal, Nucl. Phys. Proc. Suppl. 106, 586 (2002), hep-lat/0108027.

[25] E.-M. Ilgenfritz, B. Martemyanov, M. MüllerPreussker, and A. Veselov, Phys.Rev. D71, 034505 (2005), hep-lat/0412028.
[26] E.-M. Ilgenfritz, B. Martemyanov, M. MüllerPreussker, and A. Veselov, Phys.Rev. D73, 094509 (2006), hep-lat/0602002. 